\title{
Enfermedad por Coronavirus 2019, COVID-19: Aspectos a considerar en niños
}

\section{Coronavirus disease 2019, COVID-19: Aspects to consider in children}

Percy J. Sánchez-Tauma, ${ }^{1,2, a}$, Noé Atamari-Anahui ${ }^{3,4, b}$, Carlos Valera-Moreno,

\begin{abstract}
RESUMEN
La enfermedad por COVID-19 es producida por SARS-CoV-2 y actualmente es considerado como una pandemia. Presentamos este artículo de revisión con el objetivo de describir las características de esta enfermedad en los niños. Se han descrito algunas hipótesis que pueden explicar el desarrollo de esta enfermedad en niños, además de diferencias clínicas y laboratoriales a comparación de los adultos. No se ha reportado algún tratamiento en específico para este grupo poblacional. Se deben realizar estudios epidemiológicos en niños según el contexto de cada región para evaluar el comportamiento de esta enfermedad en esta población.
\end{abstract}

Palabras clave: Coronavirus; COVID-19; Niño; Síndrome Respiratorio Agudo Grave 2; Pediatría (Fuente: DeCSBIREME).

\begin{abstract}
COVID-19 disease is caused by SARS-CoV-2 and currently is considered a pandemic. We present this review article with the aim of describing the characteristics of this disease in children. Some hypotheses have been described that may explain the development of this disease in children, in addition to clinical and laboratory differences compared to adults. No specific treatment has been reported for this population group. Epidemiological studies in children should be carried out according to the context of each region to evaluate the behavior of this disease in this population.
\end{abstract}

Keywords: Coronavirus; COVID-19; Severe Acute Respiratory Syndrome Coronavirus 2; Child; Pediatrics (Source: DeCS-BIREME).

\section{INTRODUCCIÓN}

En diciembre del 2019, se reportó un grupo de casos de neumonía causados por un $B$ coronavirus recientemente identificado en la ciudad de Wuhan, provincia HubeiChina. Este coronavirus fue inicialmente denominado por la Organización Mundial de la Salud (OMS) como el nuevo coronavirus 2019 (2019-nCoV) el 12 de enero de

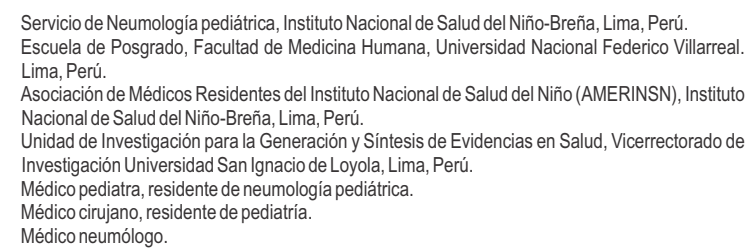

2020; posteriormente la OMS lo nombró oficialmente como enfermedad por coronavirus 2019 (COVID-19) y el grupo de estudio de coronavirus del comité internacional lo propuso nombrar como SARS-CoV-2, ambos emitidos el 11 de febrero de $2020^{(1)}$. El COVID-19 fue declarado pandemia el 11 de marzo de $2020^{(2)}$.

Los coronavirus causan principalmente infecciones del tracto respiratorio, gastrointestinal; y se clasifican en cuatro géneros principales: alphacoronavirus, betacoronavirus, gammacoronavirus, y deltacoronavirus. Los dos primeros infectan principalmente a los mamíferos; mientras que, los dos últimos infectan principalmente a las aves ${ }^{(3)}$. Entre los varios coronavirus que son patógenos para los humanos, la mayoría están asociados con síntomas clínicos leves con dos excepciones notables que han logrado ocasionar enfermedad severa, el coronavirus del Síndrome Respiratorio Agudo Severo (SARS-CoV), que surgió en Guangdong (Sur de China) en noviembre de 2002 y el coronavirus del Síndrome Respiratorio del Medio Oriente (MERS-CoV), que se detectó por primera vez en Arabia Saudita el $2012^{(4)}$.

En el presente artículo revisaremos los aspectos básicos de la enfermedad por coronavirus 2019 en los niños, debida a la importante información reciente para tomar en cuenta en la atención medica de emergencia y ambulatoria en pediatría en el contexto de esta pandemia.

\section{MATERIAL Y MÉTODOS}

Se realizó una búsqueda en la base de Pubmed que 
abarcó el periodo del 1 de enero del 2020 al 10 de mayo del 2020, empleando la siguiente estrategia de búsqueda: (("COVID 19"[Supplementary Concept] OR "severe acute respiratory syndrome coronavirus 2"[Supplementary Concept] OR "COVID-19"[tiab] OR COVID2019[tiab] OR 2019-nCoV[tiab] OR 2019nCoV[tiab] OR COVID-19[tiab] OR COVID19[tiab] OR SARS-CoV-2[tiab] OR SARS-CoV2[tiab] OR "severe acute respiratory syndrome coronavirus 2"[tiab]) OR (wuhan[All Fields] AND (coronavirus[tiab] OR "novel coronavirus"[tiab] OR "coronavirus"[MeSH Terms] OR "coronavirus infections" [mesh]))) AND ( "infant" [MeSH] OR "child" [MeSH] OR "adolescent"[MeSH] "Infant, Newborn"[MeSH] OR "Newborn"[tiab] OR "Neonate"[tiab] OR “children"[tiab] OR “Pediatrics" $[\mathrm{MeSH}])$. Para esta revision se incluyeron reportes de caso, estudios observacionales, ensayos clínicos y revisiones sistemáticas en niños (0-18 años) con COVID-19, sin excepción de idioma.

\section{Epidemiología}

En los últimos meses se han publicado reportes epidemiológicos de la infección por SARS-CoV-2, en la población infantil. Se estima que de todos los casos diagnosticados de COVID-19 en la población en general, los niños representan del 1 al $5 \%{ }^{(5)}$. Se han notificado casos en diferentes partes del mundo.

En China, un estudio retrospectivo entre el 8 de diciembre del 2019 y el 6 de febrero del 2020, reportó a 9 niños hospitalizados (edad 28 días a 1 año) con COVID19 , que no necesitaron de una unidad de cuidados intensivos o ventilación mecánica ${ }^{(6)}$. Otro estudio en China con datos de 2143 pacientes pediátricos, hasta el 8 de febrero del 2020, reportó una mediana de edad de 7 años (rango de 2 a 13), de los cuales $34,1 \%$ pacientes fueron confirmados de COVID-19 y un $65,9 \%$ como casos sospechosos $^{(7)}$. El sexo predominante en ambos grupos fue el masculino y de todos los casos confirmados el $30,5 \%$ fueron menores de 5 años $^{(7)}$. Otro estudio en China, de 1391 niños hospitalizados se ha reportado una muerte en un niño de 10 meses $^{(8)}$, y en una serie de casos de nueve pacientes pediátricos con enfermedad grave, se reportó que solo dos requirieron ventilación mecánica ${ }^{(9)}$.

En países europeos también se han registrado casos de COVID-19 en niños. En Italia, al 6 de mayo del 2020, de todos los casos de COVID-19 el 1,9\% correspondían a niños menores de 18 años (10), así mismo se registró una mortalidad del $0,2 \%$ en ese mismo grupo etario ${ }^{(11)}$. En España, al 29 de abril de todos los pacientes hospitalizados el 0,6\% correspondían a menores de 18 años ${ }^{(12)}$

En América los casos de COVID-19 en niños han ido en aumento. En Estados Unidos, entre el 12 de febrero al 2 de abril de 2020, se registró 149760 casos de COVID-19 de los cuales el 1,7\% correspondían a niños menores de 18 años, además de 3 muertes ${ }^{(13)}$. En Perú al 1 de abril del 2020 de 1323 casos de COVID-19, el 1,7\% correspondían a niños menores de 18 años. Además, al 10 de mayo se han registrado 9 niños fallecidos ${ }^{(14)}$.

\section{Patogenia}

Varios estudios han informado que el SARS-CoV-2 se une a la enzima convertidora de angiotensina 2 (ACE2), unida a la membrana del mismo modo que SARS-CoV, para obtener acceso a sus células objetivo. La proteína espiga (S) de los coronavirus facilita la entrada viral en las células objetivo. Esta proteína activa la ACE2 como receptor de entrada y emplea la serina proteasa celular TMPRSS2 para el ingreso dentro de la célula ${ }^{(15)}$. La ACE2 se expresa altamente en la cavidad oral (boca y lengua), lo que facilita la entrada viral al huésped. En los pulmones sin enfermedad, la ACE2 se expresa en las células epiteliales alveolares tipo I y II. Después de la infección, la entrada de SARS-CoV-2 comienza con la unión de la proteína $S$ a la ACE2 en la superficie alveolar. La unión de SARS-CoV-2 a ACE2 estimula la endocitosis de todo el complejo SARS-CoV-2/ACE2, induciendo la fusión de la membrana celular y el virus, formándose de esta manera endosomas en el citoplasma, facilitado por un $\mathrm{pH}$ ácido. Una vez dentro de las células, el SARS-CoV2 inicia la transcripción endógena de las células alveolares para replicarse y propagarse por todo el pulmón ${ }^{(16)}$.

\section{Consideraciones en niños}

Los niños tienen un sistema de respuesta inmune especial que es diferente de los adultos; por lo tanto, los pacientes pediátricos con COVID-19 tienen sus propias características clínicas y respuestas terapéuticas. Sin embargo, existe la pregunta ¿por qué en la población pediátrica no hay elevada mortalidad comparado con los adultos? Se han consideramos tres teorías al respecto:

a. La proteína ACE2 está ampliamente distribuida en el cuerpo, y la baja susceptibilidad de los niños al SARS-CoV-2 está relacionada con el desarrollo y la función de la proteína ACE2, como la baja capacidad de unión de esta con el virus. Además, la respuesta intracelular inducida por ACE2 en las células epiteliales alveolares en los niños es menor que en los adultos ${ }^{(17)}$.

b. En los niños existe mayor cantidad de infecciones vírales por lo que tienen mayor cantidad de anticuerpos contra virus respiratorios que los adultos. Esto produciría que la infección sea leve más aún en lugares donde existe mayor cantidad de infectados y coincide con temporadas de invierno donde circulan mayor cantidad de virus respiratorios. Además la presencia de otros virus simultáneos en la mucosa de los pulmones y de las vías respiratorias, que son comunes en niños 
pequeños, podrían limitar el crecimiento de SARSCoV-2 por interacciones directas de virus a virus, es decir por competencia ${ }^{(18)}$.

c. En los casos confirmados de adultos en Wuhan se observó una disminución significativa o progresiva en el número absoluto de linfocitos en sangre periférica en la etapa temprana de la enfermedad, y los subconjuntos de linfocitos $T$ muestran una disminución de células T CD4 + y CD8 +, lo que sugiere que SARS-CoV-2 puede disminuir linfocitos siendo ser una razón importante para que el virus pueda proliferar y propagarse en las primeras etapas de la enfermedad. Los casos graves generalmente progresan de 7 a 10 días después del inicio de la enfermedad; esto sugiere que la inmunidad adaptativa está involucrada en el daño inmunológico durante la progresión de la enfermedad. Sin embargo, en los niños el recuento de glóbulos blancos y el número absoluto de linfocitos son en su mayoría normales, y no se ha producido ningún agotamiento de los linfocitos; este hallazgo puede estar relacionado con la imperfección del desarrollo inmune natural conduciendo a un bajo nivel de respuesta inmune adaptativa posterior, que parece explicar las manifestaciones clínicas leves de la enfermedad infantil ${ }^{(17)}$; incluso en población pediátrica trasplantada que usa terapia inmunosupresora, como lo reportado en Italia ${ }^{(19)}$.

\section{Manifestaciones Clínicas}

Según los datos epidemiológicos actuales, el período de incubación del COVID-19 varía de 1 a 14 días. Según las condiciones actuales de los casos reportados, los niños pertenecen principalmente a casos de conglomerados familiares $^{(20)}$. La mayoría de ellos tienen buen pronóstico y en casos leves se recuperan de 1 a 2 semanas después del inicio de la enfermedad ${ }^{(7)}$.

Existen aún limitados reportes de COVID-19 en recién nacidos y lactantes. En China, se ha reportado un recién nacido con COVID-19 con síntomas leves de tos, rinorrea y dificultad respiratoria ${ }^{(21)}$; y otro de 19 días de vida que tuvo como motivo de consulta a emergencia y como primera manifestación clínica a los vómitos y deposiciones liquidas sin moco y sin sangre (4-5 veces/día), seguido de episodios febriles ${ }^{(22)}$. Asimismo, se han casos severos en ese grupo de edad. En una paciente de 13 meses, se inició el cuadro clínico con diarrea intermitente y vómitos por 6 días que progresivamente evolucionó con fiebre y dificultad respiratoria, fue internado en la unidad de cuidados intensivos neonatal por neumonía por COVID-19 asociado a shock séptico e insuficiencia renal ${ }^{(23)}$.

Un estudio en China en 171 niños con COVID-19, reportó una edad promedio de 6,7 años ${ }^{(8)}$, siendo las manifestaciones clínicas más frecuentes la tos, el eritema faríngeo y la fiebre (Tabla №1). Asimismo, 27 pacientes $(15,8 \%)$ no presentaron síntomas de infección ni características radiológicas de neumonía y 12 pacientes tenían características radiológicas de neumonía; sin embargo, no presentaban síntomas de infección ${ }^{(8)}$. En otro reporte de 36 pacientes con una media de edad de 8,3 años se encontró a la fiebre y tos como principales manifestaciones clínicas ${ }^{(20)}$.

En Estados Unidos, la fiebre, la tos y la cefalea fueron los síntomas más frecuente hallados en pacientes menores de 18 años $^{(13)}$; sin embargo, en dos pacientes lactantes (uno de 25 días y otro de 56 días) se ha descrito solo fiebre con ausencia de dificultad respiratoria requiriendo hospitalización ${ }^{(24)}$. En Italia, en pacientes pediátricos que llegaban a emergencia se encontró a la tos y la falta de alimentación como síntomas predominantes ${ }^{(25)}$.

En pacientes que han desarrollado enfermedad severa por COVID-19, también se han descrito síntomas. En Wuhan-China, de ocho pacientes pediátricos con enfermedad severa encontraron a la polipnea, fiebre, tos, expectoración, náuseas y vómitos como síntomas más frecuentes ${ }^{(9)}$; de todos ellos la polipnea según los autores debe destacarse en pacientes pediátricos graves, incluso sin fiebre ${ }^{(9)}$.

Se han reportado también manifestaciones neurológicas en niños. En Estados Unidos se ha reportado un caso de encefalitis por COVID-19 en un niño de 11 años, que inició con debilidad generalizada, sin evidencia de síntomas respiratorios, ni síntomas febriles ${ }^{(26)}$. También se han registrado manifestaciones cutáneas aisladas en niños como lesiones eritematosas papulares a predominio de manos y $\operatorname{codos}^{(27)}$.

\section{DIAGNÓSTICO}

Debido a las diferentes manifestaciones clínicas en menores de 18 años, puede estar justificado la realización de pruebas de tamizaje de COVID-19 a los pacientes lactantes cuando estos se hospitalizan, pues se ha descrito en ocasiones solo síntomas febriles (24). También se recomienda la detección de otros gérmenes respiratorios según el contexto epidemiológico de cada región, pues se ha reportado coinfección de COVID-19 con otros virus respiratorios comunes ${ }^{(28)}$.

El desarrollo de anticuerpos tipo lgM se produce a los 5 a 7 días; mientras que, los anticuerpos tipo IgG a los 15 a 21 días $^{(29)}$, por lo que para el diagnóstico de COVID-19 se recomienda la realización de pruebas rápidas para detectar anticuerpos IgM/lgG contra SARS-CoV-2 o una prueba de reacción de cadena de la polimerasa con transcriptasa reversa (RT-PCR). Si es positiva la prueba rápida se considera como caso confirmado, pero si es negativa se recomienda la realización de una segunda prueba para los casos leves a la semana de la primera 
Tabla N¹. Manifestaciones clínicas en pacientes pediátricos infectados por SARS-CoV-2.

\begin{tabular}{|c|c|c|c|c|}
\hline País & Periodo de estudio & Edad (años) & $\begin{array}{c}\text { Número de } \\
\text { participantes }\end{array}$ & Características Clínicas \\
\hline China(8) & $\begin{array}{c}28 \text { enero-26 } \\
\text { febrero }\end{array}$ & $6,7(0-15)^{*}$ & 171 & $\begin{array}{l}\text { Tos }(48,5 \%) \text {, eritema faríngeo }(46,2 \%) \text {, fiebre }(41,5 \%) \text {, diarrea } \\
(8,8 \%) \text {, fatiga }(7,6 \%) \text {, rinorrea }(7,6 \%) \text {, vómitos }(6,4 \%) \text {, diarrea } \\
(8,8 \%) \text {, taquipnea al ingreso }(28,7 \%) \text {, saturación oxigeno }<92 \%(2,3 \%)\end{array}$ \\
\hline Estados Unidos (13) & 12 febrero-2 abril & $11(0 \text { a } 17)^{*}$ & 2572 & $\begin{array}{l}\text { (+) Fiebre }(56 \%) \text {, tos }(54 \%) \text {, dificultad para respirar }(13 \%) \text {, mialgia } \\
(23 \%) \text {, dolor de garanta }(24 \%) \text {, náuseas y vómitos }(11 \%) \text {, dolor } \\
\text { abdominal }(5,8 \%) \text {, diarrea }(13 \%) \text {, cefalea }(28 \%) \text {. }\end{array}$ \\
\hline China(20) & 17 enero-1 marzo & $8,3(+/-3,5)^{* *}$ & 36 & Fiebre $(36 \%)$, tos seca $(19 \%)$. \\
\hline Italia(25) & 3 marzo-27 marzo & $3,3(0-17,5)^{*}$ & 100 & $\begin{array}{l}\text { Tos }(44 \%) \text {, falta de alimentación o dificultad para alimentarse }(23 \%) \text {, } \\
\text { rinorrea }(22 \%) \text { dificultad para respirar }(11 \%) \text {, náuseas y vómitos } \\
(10 \%) \text {. Diarrea }(9 \%) \text {, dolor abdominal }(4 \%) .\end{array}$ \\
\hline
\end{tabular}

Tabla N². Interpretación de resultado de las pruebas rápidas (IgM/lgM) y RT-PCR para COVID-19.

\begin{tabular}{cccl} 
& \multicolumn{2}{l}{ Resultado } & \multicolumn{2}{l}{ Interpretación } \\
\cline { 1 - 2 } RT-PCR & IgM & IgG & \\
\hline- & - & - & Negativo / falso negativo RT-PCR en etapa temprana \\
+ & - & - & Etapa inicial de la infección (periodo de ventana) \\
+ & + & - & Etapa temprana / infección aguda \\
+ & + & + & Infección activa (transición vs reinfección) \\
+ & - & - & Etapa tardía de la infección \\
- & + & + & Estadio temprano / falso positivo serológico / falso negativo RT-PCR \\
- & - & + & Enfección pasada / falso negativo RT-PCR \\
- & + & &
\end{tabular}

Fuente: Adaptado de la Asociación Española de Pediatría de Atención Primaria 2020

prueba. Con respecto al RT-PCR, esta debe ser tomada del aparato respiratorio superior mediante hisopado nasofaríngeo; si sale positivo se considera como caso confirmado y si sale negativo y la evolución clínica es desfavorable se recomienda la realización de una segunda prueba. La interpretación de estos resultados se describe en la tabla $\mathrm{N}^{\circ} 2$.

\section{Hallazgo de imágenes}

Radiografía de tórax. - En la etapa inicial de los casos de neumonía las imágenes del tórax muestran múltiples sombras pequeñas e irregulares y cambios intersticiales, notables en la periferia pulmonar. Los casos graves pueden desarrollarse aún más hasta opacidad bilateral de vidrio esmerilado múltiple, sombras infiltrantes y consolidación pulmonar, con derrame pleural infrecuente $\mathrm{e}^{(30)}$.

Tomografía de tórax. - Se ha encontrado lesiones típicas en este grupo etario a las opacidades de vidrio esmerilado subpleurales unilaterales o bilaterales $(60 \%)$, y a las consolidaciones con signo del halo
$(50 \%)^{(31)}$. Otro reporte en China encontró opacidades de vidrio esmerilado $32 \%$, sombreado local irregular $(18,7 \%)$, sombreado parcheado bilateral $(12,3 \%)$ y anomalías intersticiales en $1,2 \%^{(8)}$.

Ecografía de tórax. - Un estudio de 8 pacientes pediátricos de Italia, en la ecografía pulmonar se encontró consolidación en 2 pacientes y patrón de líneas $B$ intersticiales en 5 ; estos últimos fueron más destacados en casos moderados y severos ${ }^{(32)}$.

\section{Hallazgos laboratoriales}

En un estudio de 171 niños en China, se ha reportado un recuento de leucocitos $<5,5 \times 109 / \mathrm{L}$ en el $26 \%$ y linfopenia (recuento de linfocitos, $<1,2 \times 109 / \mathrm{L}$ ) en el $3,5 \%{ }^{(8)}$. Asimismo, la procalcitonina se elevó $(>46 \mathrm{pg} / \mathrm{ml})$ en el $64 \%$, la proteína C reactiva ( $>10 \mathrm{mg} / \mathrm{L}$ ) en el $19,7 \%$, la aspartato aminotransferasa ( $>50 \mathrm{U} / \mathrm{L}$ ) en el $14,6 \%$ y el dímero $\mathrm{D}(>0,6 \mathrm{mg} / \mathrm{LFEU})$ en el $14,1 \%{ }^{(8)}$. Otra revisión de 12 estudios al 5 de marzo 2020 que incluyó a 66 pacientes pediátricos, encontró diferencias con respecto al número de leucocitos: recuento normal en 
$69,6 \%$, recuento aumentado y a la vez disminuido $15,2 \%{ }^{(33)}$. Con respecto a los neutrófilos, la mayoría tuvo recuentos normales, el $4,6 \%$ por encima y un $6 \%$ por debajo del rango normal ${ }^{(33)}$. Solo el $3 \%$ de lactantes con enfermedad leve a moderada se encontraron con linfopenia y la elevación de la proteína $\mathrm{C}$ reactiva y la procalcitonina se elevó en $13,6 \%$ y $10,6 \%$ de los $\operatorname{casos}^{(33)}$.

\section{Severidad}

La tasa de hospitalización en niños con COVID-19 varía del 6 al 20\%, y la de hospitalización en cuidado intensivos de 0,5 al $2 \%{ }^{(13)}$. Los pacientes con ciertas comorbilidades tienen mayor riesgo de llegar a la hospitalización como casos graves. En Estados Unidos de 345 niños con COVID-19, el 23\% tenía alguna comorbilidad dentro de las que destaca el asma 11,5\%, enfermedad cardiovascular 7,2\% e inmunosupresión $2,8 \%{ }^{(13)}$; todos estos últimos llegaron a hospitalización. Un estudio en China de 171 niños reportó que 3 pacientes requirieron cuidados intensivos y ventilación mecánica, todos ellos tenían alguna comorbilidad (hidronefrosis, leucemia e invaginación intestinal) ${ }^{(8)}$. Otro reporte en China de 728 pacientes pediátricos con COVID-19, encontró como casos asintomáticos al 13\%, leve al $43 \%$, moderada al $41 \%$, grave al $2,5 \%$ y crítico al $0,4 \%(11 \% \text { en menores de un año })^{(7)}$. Esta clasificación de severidad fue reportada en base a las recomendaciones para el diagnóstico, prevención y control de la nueva infección por coronavirus 2019 en niños ${ }^{(34)}$, que los clasifica como:

a. Infección asintomática. - cuando no se presenta ningún síntoma ni signo clínico y la imagen del tórax es normal, mientras que la prueba de ácido nucleico 2019-nCoV sea positivo.

b. Leve. - cuando se evidencia síntomas de infección aguda del tracto respiratorio superior y al examen físico muestra congestión de la faringe, pero sin anormalidades en la auscultación. Sin embargo, algunos casos pueden no tener fiebre o solo tener síntomas digestivos náuseas, vómitos, dolor abdominal y diarrea.

c. Moderado. - cuando se evidencia neumonía, fiebre y tos frecuente (seca o productiva), con episodios de sibilancias, estertores secos $\mathrm{y} / \mathrm{o}$ estertores húmedos a la auscultación, pero sin llegar a la hipoxemia. Algunos casos pueden no tener signos y síntomas clínicos, pero la tomografía de tórax muestra lesiones pulmonares que son subclínicas.

d. Grave. - cuando la enfermedad generalmente progresa alrededor de una semana y produce disnea con cianosis central. La saturación de oxígeno es inferior al $92 \%$, con otras manifestaciones de hipoxia.

e. Crítico. - cuando los niños pueden progresar rápidamente al SARS o insuficiencia respiratoria, y pueden tener shock, encefalopatía, lesión miocárdica o insuficiencia cardíaca, disfunción de la coagulación y lesión renal aguda.

\section{Manejo y tratamiento}

Como la mayoría de los síntomas son de vías respiratorias en niños, se recomienda evitar el examen de la orofaringe a menos que sea muy necesario. Si se realiza este procedimiento se debe usar equipo de protección personal así el niño no tenga sintomatología. No existe un tratamiento antiviral específico recomendado para COVID-19 en niños, y actualmente no hay vacuna disponible. El tratamiento es sintomático y la oxigenoterapia representa la principal intervención de tratamiento para pacientes con infección grave. La ventilación mecánica puede ser necesaria en casos de insuficiencia respiratoria refractaria a la oxigenoterapia, mientras que el soporte hemodinámico es esencial para controlar el shock séptico.

A pesar de ello, se ha reportado algunos manejos específicos utilizados en algunos reportes publicados como los siguientes:

- Recién nacido con diagnostico a las 36 horas de infección por SARS-CoV-2, recibió monitoreo continuo de funciones vitales, no requirió soporte oxigenatorio por lo que no se le dio tratamiento especial siendo dado de alta a los 15 días del nacimiento ${ }^{(21)}$.

- Neonato de 19 días, quien como manifestación inicial presentó vómitos y diarrea, recibió tratamiento sintomático con interferón aerosol nasal y reposición de líquidos, con mejoría clínica y fue dado de alta después de 14 días $^{(22)}$.

- Gemelas de un año, hospitalizadas por presentar fiebre, tos y secreción nasal (gemela 1), recibió gammaglobulina endovenosa una vez por día por 3 días, interferón alfa $2 B$ en aerosol una pulverización cada 3 horas por 2 días y atomización con interferón 2 veces al día por 5 días. La gemela 2 recibió el mismo tratamiento que la gemela 1 excepto la gammaglobulina. Ambas mejoraron clínicamente y salieron de alta luego de 9 días de hospitalización sin complicaciones ${ }^{(35)}$.

- El primer caso de infección por SARS-CoV-2 en Corea, fue de una niña de 10 años sin enfermedad subyacente; se presentó a emergencia subfebril sin disnea ni cianosis. La tomografía mostró consolidaciones irregulares o nodulares con opacidades de vidrio esmerilado periféricas en áreas subpleurales del lóbulo inferior derecho; sin embargo, no requirió terapia antiviral solo manejo de soporte ${ }^{(36)}$.

- El primer caso de neumonía grave por SARS-CoV-2 en China, en un niño de 1 año y 1 mes; debutó con vómitos y diarreas de 5 días de evolución, presentando luego distrés respiratorio. Se realizó tomografía de tórax observándose imagen en vidrio esmerilado y zonas de condensación en parénquima pulmonar derecho, por ese motivo es hospitalizado en cuidados intensivos recibiendo ventilación 
mecánica e inotrópicos, además de nebulización con interferón, gammaglobulina endovenosa, oseltamivir y metilprednisolona. Al décimo día después del ingreso, la condición del paciente mejoró significativamente, la saturación de oxígeno se mantuvo por encima de $95 \%$ siendo extubado y recibiendo tratamiento sintomático hasta el alta ${ }^{(23)}$.

- En un estudio en China de 36 pacientes pediátricos la terapia fue con interferón alfa por aerosol dos veces al día, jarabe de lopinavir-ritonavir dos veces al día y oxigenoterapia. Todo ello desde casos leves a moderados $^{(20)}$.

Como hemos podido observar, aún la evidencia es limitada respecto al tratamiento de COVID-19 en pediatría. Es posible que en algunos casos en que se usaron tratamiento, éstos podrían haber sido innecesarios e incluso hasta dañinos pues la recuperación en algunos niños fue incluso solo con tratamiento sintomático, observación y aislamiento, salvo en formas graves que requieren de medidas de soporte ventilatorio; así como, ocurre en otras enfermedades pediátricas que producen insuficiencia respiratoria.

La enfermedad por Coronavirus 2019, COVID-19 se ha incrementado en la población general a partir del primer caso confirmado en China. Los estudios epidemiológicos indican que esta pandemia se irá incrementando en la población pediátrica. Es importante para el médico general y médico pediatra tener en cuenta algunas características recientemente reportadas en la población infantil para un diagnóstico oportuno y manejo adecuado, evitando de esta manera el contagio y complicaciones propias de esta enfermedad. Asimismo, se recomienda el desarrollo de estudios clínico-epidemiológicos que ayuden a conocer mejor el comportamiento de esta enfermedad, especialmente en la población infantil; que, a pesar de tener una mortalidad disminuida, encabeza la mayor cantidad de consultas en la atención primaria.

Conflictos de interés: Los autores niegan conflictos de interés.

Financiamiento: Autofinanciado.

Contribuciones de autoría: PJST y NAA participaron en la concepción y diseño del artículo. Todos los autores participaron en la interpretación de datos, revisión crítica del artículo, recolección de datos, redacción y aprobación de la versión final.

\section{REFERENCIAS BIBLIOGRÁFICAS}

1. Guo Y-R, Cao Q-D, Hong Z-S, Tan Y-Y, Chen S-D, Jin $\mathrm{H}-\mathrm{J}$, et al. The origin, transmission and clinical therapies on coronavirus disease 2019 (COVID-19) outbreak - an update on the status. Mil Med Res. 2020;7(1):11.
2. World Health Organization (WHO). WHO DirectorGeneral's opening remarks at the media briefing on COVID-19 - 11 March 2020 [Internet]. 2020 [citado 10 de mayo de 2020]. Disponible en: https://www.who.int/dg/speeches/detail/whodirector-general-s-opening-remarks-at-themedia-briefing-on-covid-19---11-march-2020

3. Tang Q, Song Y, Shi M, Cheng Y, Zhang W, Xia X-Q. Inferring the hosts of coronavirus using dual statistical models based on nucleotide composition. Sci Rep. 2015;5(1):1-8.

4. Lu R, Zhao X, Li J, Niu P, Yang B, Wu H, et al. Genomic characterization and epidemiology of 2019 novel coronavirus: implications for virus origins and receptor binding. The Lancet. 2020;395(10224):565-74.

5. Ludvigsson JF. Systematic review of COVID-19 in children shows milder cases and a better prognosis than adults. Acta Paediatr. 2020;109(6):1088-95.

6. Wei M, Yuan J, Liu Y, Fu T, Yu X, Zhang Z-J. Novel Coronavirus Infection in Hospitalized Infants Under 1 Year of Age in China. JAMA [Internet]. 2020 [citado 10 de mayo de 2020]; Disponible en: https://jamanetwork.com/journals/jama/fullar ticle/2761659

7. Dong $Y$, Mo X, Hu Y, Qi X, Jiang F, Jiang Z, et al. Epidemiology of COVID-19 Among Children in China. Pediatrics. 2020.

8. Lu X, Zhang L, Du H, Zhang J, Li YY, Qu J, et al. SARS-CoV-2 Infection in Children. N Engl J Med. 2020.

9. Sun D, Li H, Lu X-X, Xiao H, Ren J, Zhang F-R, et al. Clinical features of severe pediatric patients with coronavirus disease 2019 in Wuhan: a single center's observational study. World J Pediatr. 2020.

10. NYC Health. Italy: coronavirus cases by age 2020 [Internet]. Statista. 2020 [citado 10 de mayo de $\left.\begin{array}{llll}2 & 0 & 2 & 0\end{array}\right]$. D i s p o n i b l e e n : https://www.statista.com/statistics/1103023/c oronavirus-cases-distribution-by-age-groupitaly/

11. NYC Health. Italy: coronavirus death rate by age [Internet]. Statista. 2020 [citado 10 de mayo de $\left.\begin{array}{llll}2 & 0 & 2 & 0\end{array}\right]$. D is p on ible e n : https: / /www.statista.com/statistics/1106372/c oronavirus-death-rate-by-age-group-italy/

12. NYC Health. Spain: coronavirus hospital case distribution by age 2020 [Internet]. Statista. 2020 [citado 10 de mayo de 2020]. Disponible en: https: / /www.statista.com/statistics/1106425/c ovid-19-mortality-rate-by-age-group-in-spainmarch/

13. CDC COVID-19 Response Team. Coronavirus Disease 2019 in Children - United States, February 12-April 2, 2020. MMWR Morb Mortal Wkly Rep. 2020;69(14):422-6.

14. Ministerio de Salud del Perú. Sala Situacional COVID-19 Perú [Internet]. 2020 [citado 10 de mayo 
d e $\left.\begin{array}{lllll}2 & 0 & 2 & 0\end{array}\right]$. Dispon ible e $n$ : https://www.minsa.gob.pe/covid19/sala_situaci onal.asp

15. Hoffmann M, Kleine-Weber H, Schroeder S, Krüger $\mathrm{N}$, Herrler T, Erichsen S, et al. SARS-CoV-2 Cell Entry Depends on ACE2 and TMPRSS2 and Is Blocked by a Clinically Proven Protease Inhibitor. Cell. 2020.

16. Perico L, Benigni A, Remuzzi G. Should COVID-19 Concern Nephrologists? Why and to What Extent? The Emerging Impasse of Angiotensin Blockade. Nephron. 2020;1-9.

17. Fang F, Luo XP. Facing the pandemic of 2019 novel coronavirus infections: the pediatric perspectives. Chin J Pediatr. 2020;58(2):81-5.

18. Brodin P. Why is COVID-19 so mild in children? Acta Paediatr. 2020.

19. D'Antiga L. Coronaviruses and immunosuppressed patients. The facts during the third epidemic. Liver Transplant. 2020.

20. Qiu H, Wu J, Hong L, Luo Y, Song Q, Chen D. Clinical and epidemiological features of 36 children with coronavirus disease 2019 (COVID-19) in Zhejiang, China: an observational cohort study. Lancet Infect Dis. 2020.

21. Wang S, Guo L, Chen L, Liu W, Cao Y, Zhang J, et al. A case report of neonatal COVID-19 infection in China. Clin Infect Dis. 2020.

22. Wang J, Wang D, Chen G-C, Tao X-W, Zeng L-K. SARS-CoV-2 infection with gastrointestinal symptoms as the first manifestation in a neonate. Chin J Contemp Pediatr. 2020;22(3):211-4.

23. Chen F, Liu ZS, Zhang FR, Xiong RH, Chen Y, Cheng $X F$, et al. First case of severe childhood novel coronavirus pneumonia in China. Chin J Pediatr. 2020;58(0):E005.

24. Paret M, Lighter J, Pellett Madan R, Raabe VN, Shust GF, Ratner AJ. SARS-CoV-2 infection (COVID19) in febrile infants without respiratory distress. Clin Infect Dis. 2020.

25. Parri N, Lenge $M$, Buonsenso $D$. Children with Covid-19 in Pediatric Emergency Departments in Italy. N Engl J Med. 2020.

26. McAbee GN, Brosgol Y, Pavlakis S, Agha R, Gaffoor M. Encephalitis Associated with COVID-19 Infection in an 11 Year-Old Child. Pediatr Neurol. 2020.

27. Recalcati S, Barbagallo T, Frasin LA, Prestinari F,
Cogliardi A, Provero MC, et al. Acral cutaneous lesions in the Time of COVID-19. J Eur Acad Dermatol Venereol. 2020.

28. Xia W, Shao J, Guo Y, Peng X, Li Z, Hu D. Clinical and $\mathrm{CT}$ features in pediatric patients with COVID19 infection: Different points from adults. Pediatr Pulmonol. 2020;55(5):1169-74.

29. Guo L, Ren L, Yang S, Xiao M, Chang D, Yang F, et al. Profiling Early Humoral Response to Diagnose Novel Coronavirus Disease (COVID-19). Clin Infect Dis. 2020.

30. Chen Z-M, Fu J-F, Shu Q, Chen Y-H, Hua C-Z, Li F-B, et al. Diagnosis and treatment recommendations for pediatric respiratory infection caused by the 2019 novel coronavirus. World J Pediatr. 2020.

31. Xia W, Shao J, Guo Y, Peng X, Li Z, Hu D. Clinical and CT features in pediatric patients with COVID19 infection: Different points from adults. Pediatr Pulmonol. 2020.

32. Denina M, Scolfaro C, Silvestro E, Pruccoli G, Mignone $\mathrm{F}$, Zoppo $\mathrm{M}$, et al. Lung Ultrasound in Children With COVID-19. Pediatrics. 2020.

33. Henry BM, Lippi G, Plebani M. Laboratory abnormalities in children with novel coronavirus disease 2019. Clin Chem Lab Med. 2020.

34. Society of Pediatrics, Chinese Medical Association, Editorial Board, Chinese Journal of Pediatrics. Recommendations for the diagnosis, prevention and control of the 2019 novel coronavirus infection in children (first interim edition). Chin J Pediatr. 2020;58(0):E004.

35. Zhang $G-X$, Zhang A-M, Huang L, Cheng L-Y, Liu Z-X, Peng $X-L$, et al. Twin girls infected with SARS-CoV2. Chin J Contemp Pediatr. 2020;22(3):221-5.

36. Park JY, Han MS, Park KU, Kim JY, Choi EH. First Pediatric Case of Coronavirus Disease 2019 in Korea. J Korean Med Sci. 2020;35(11)

\section{Correspondencia}

Noé Atamari-Anahui

Dirección: Avenida La Fontana 550 La Molina Lima, Perú.

Teléfono: (+51) 984140761

Correo: noe.atamari@gmail.com

\section{Revisión de pares}

Recibido: 15/03/2020

Aceptado: 30/03/2020 